\title{
Mass spectrometry hybridized with gas-phase InfraRed spectroscopy for glycan sequencing
}

DOI:

10.1016/j.sbi.2019.12.014

\section{Document Version}

Accepted author manuscript

Link to publication record in Manchester Research Explorer

\section{Citation for published version (APA):}

Gray, C. J., Compagnon, I., \& Flitsch, S. L. (2020). Mass spectrometry hybridized with gas-phase InfraRed spectroscopy for glycan sequencing. Current Opinion in Structural Biology, 62, 121-131.

https://doi.org/10.1016/j.sbi.2019.12.014

\section{Published in:}

Current Opinion in Structural Biology

\section{Citing this paper}

Please note that where the full-text provided on Manchester Research Explorer is the Author Accepted Manuscript or Proof version this may differ from the final Published version. If citing, it is advised that you check and use the publisher's definitive version.

\section{General rights}

Copyright and moral rights for the publications made accessible in the Research Explorer are retained by the authors and/or other copyright owners and it is a condition of accessing publications that users recognise and abide by the legal requirements associated with these rights.

\section{Takedown policy}

If you believe that this document breaches copyright please refer to the University of Manchester's Takedown Procedures [http://man.ac.uk/04Y6Bo] or contact uml.scholarlycommunications@manchester.ac.uk providing relevant details, so we can investigate your claim.

\section{OPEN ACCESS}




\title{
Mass spectrometry hybridized with gas-phase InfraRed spectroscopy for glycan sequencing
}

\author{
Gray $C J,{ }^{1}$ Compagnon ${ }^{2}$ and Flitsch $S L^{1}$
}

1 School of Chemistry \& Manchester Institute of Biotechnology, The University of Manchester, 131

Princess Street, Manchester M1 7DN, UK

2 Univ. Lyon, Université Claude Bernard Lyon 1, CNRS, Institut Lumière Matière, F-69622

Villeurbanne, France.

Corresponding Authors: Sabine Flitsch (Sabine.Flitsch@manchester.ac.uk) and Isabelle Compagnon (isabelle.compagnon@univ-lyon1.fr)

\section{Abstract}

Precise structural differentiation of often isomeric glycans is important given their roles in numerous biological processes. Mass spectrometry (MS) (and tandem MS) is one of the analytical techniques at the forefront of glycan analysis given its speed, sensitivity in producing structural information as well as the fact it can be coupled to other orthogonal analytical techniques such as liquid chromatography (LC) and ion mobility spectrometry (IMS). This review describes another family of techniques that are more commonly being hybridized to MS(/MS) namely gas-phase infrared (IR) spectroscopy, whose rise is in part due to the development and improved accessibility of tunable IR lasers. Gas-phase IR can often differentiate fine isomeric differences ubiquitous within carbohydrates that MS may be 'blind' to. There are also examples of cryogenic gas-phase IR spectroscopy with much greater spectral resolution as well as hybridizing with separative methods (LC, IMS). Furthermore, collision-induced dissociation (CID) product ions can also be probed by IR, which may be beneficial to deconvolute spectra, aid analysis and build spectral libraries, thus generating novel opportunities for fragment-based approaches to analyse glycans.

\section{Introduction}

Glycans mediate numerous biological events including host-pathogen interactions, cell differentiation, motility and intercellular and intracellular signalling; many of these through regio-/stereo-specific recognition of glycan epitopes with glycan-binding proteins (GBPs) .[1,2] Additionally, aberrant or truncated glycoforms are indicative of disease states including cancer (increased branching of $\mathrm{N}$-glycans, higher density of O-glycans, truncated structures and prominent sialic acid and fucose terminating species),[3] muscular dystrophies (loss of O-mannose $\beta 1,2-\mathrm{N}$ acetylglucosaminyltranferase activity inhibiting O-mannosyl glycan synthesis),[4] as well as other congenital disorders of glycosylation (CDGs) (various and sometimes cumulative changes ranging from issues with glycan assembly and transfer, glycan processing, hyperglycosylation).[5] These changes are associated with dysregulation of cellular cycles and disease progression. As a result, there is an exciting opportunity to develop glycan-based therapies to treat diseases. Knowledge of their precise atomistic structure, as well as their interactome, (beyond the scope of this 
review) facilitate design of treatments (e.g. glycan mimetics, glycosyltransferase inhibitors or glycan-based vaccines) and are therefore crucially important.[3,6]

(a)

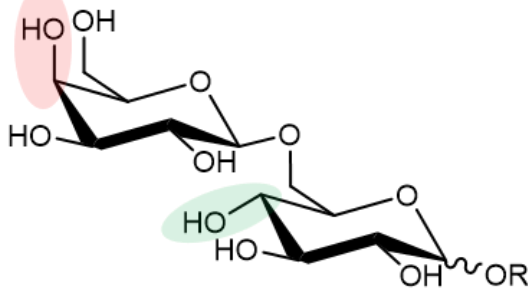

Galp $\beta 1,6 \mathrm{Glcp}-\mathrm{R}$

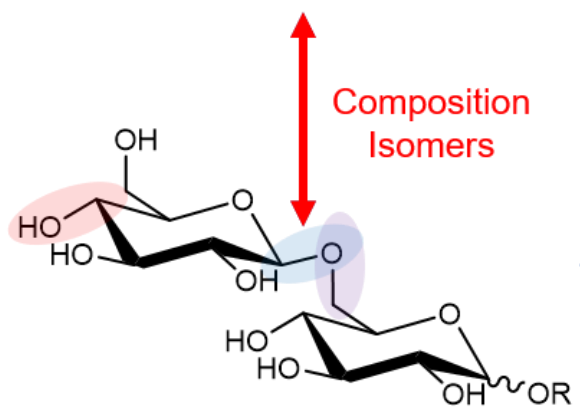

Glcpß1,6Glcp-R

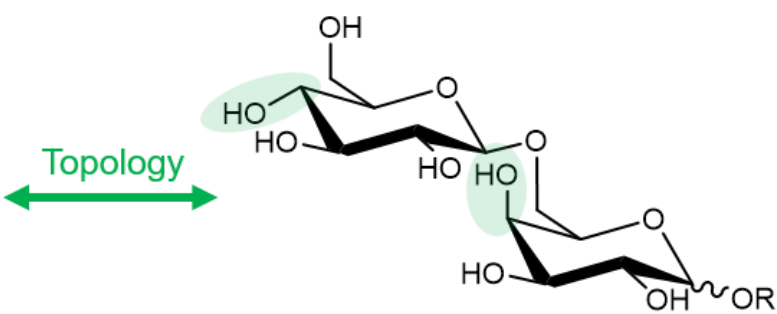

GIcp $\beta 1,6$ Galp-R
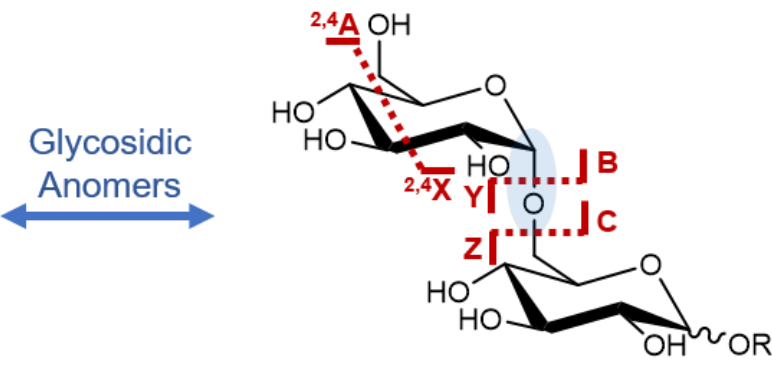

Glcpa1,6Glcp-R

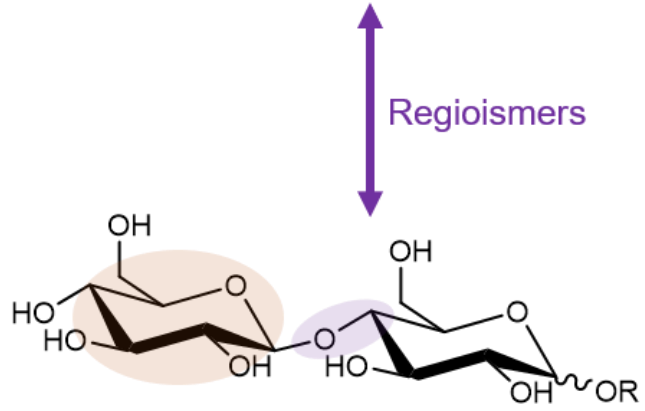

Glcpß1,4Glcp-R

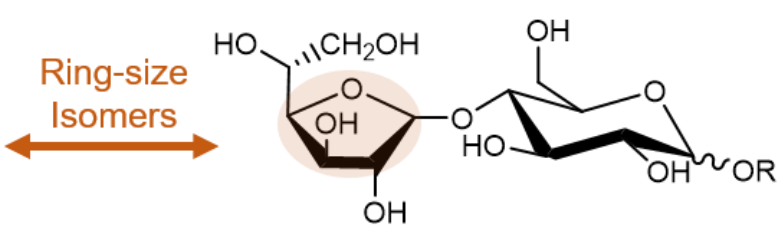

Glcfß1,4Glcp-R
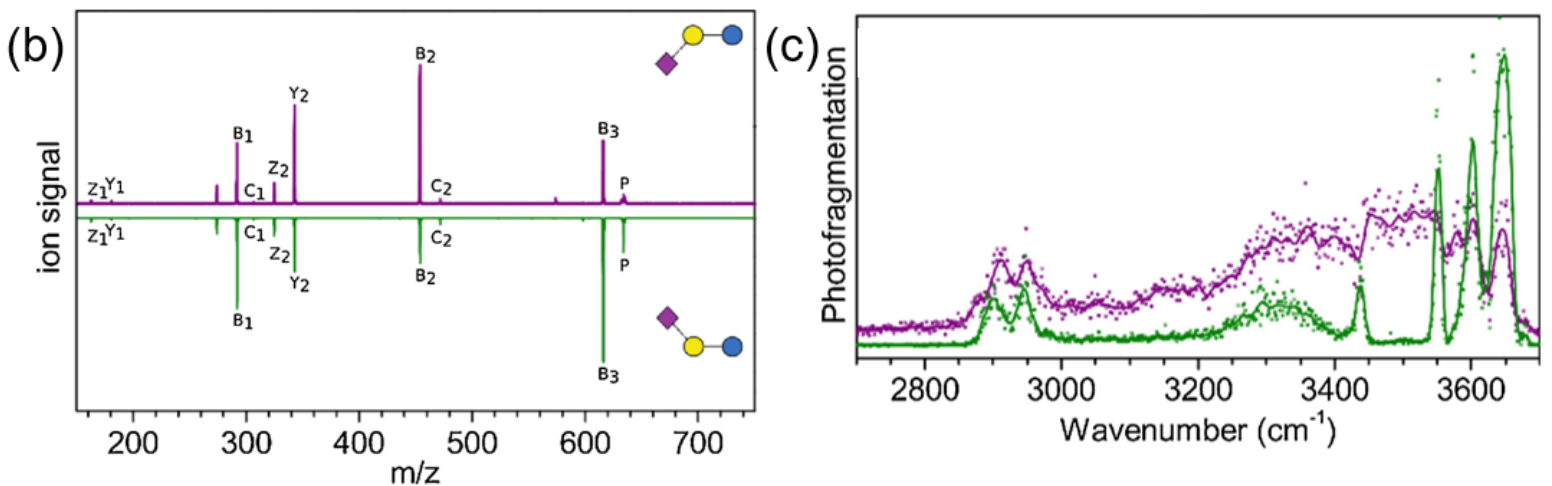

Figure 1. - The numerous families of isomers that can be found in glycans (a). Within this scheme, the dissociation nomenclature proposed by Domon-Costello[7] for glycosidic (B-, C-, Z- and Y-ion) and cross-ring fragmentation ( $\mathrm{X}$ - and $\mathrm{A}$-ions, where the numbers in superscript refer to the which bonds are cleaved in the ring) is shown, which is referred to during this Opinion. Also depicted are MS/MS spectra of isomeric trisaccharides (b) Neu5Aca2,3Galß1,4Glc (top, purple) and Neu5Aca2,6Galß1,4Glc (bottom, green) showing that the product ions formed are identical with only slight differences in intensities being observed. However, these trisaccharides produce unique gasphase IR fingerprints (c). [reproduced with permission from Ref 8] Trisaccharide structures are shown in representative SNFG nomenclature.[9] 
Despite this, their unambiguous characterization is extremely challenging, especially for less studied systems such as plant glycosides and bacterial glycans. This derives from: the numerous, often isomeric, monosaccharides (Glucose, Glc, Mannose, Man and Galactose, Gal as well as N-acetylglucosamine (GlcNAc) and Nacetylgalactosamine, GalNAc) that can exist in different ring sizes (overwhelmingly structures are found to be pyranose, $p$, but furanose, $f$, isomers exist); multiple linkage positions that can form in different stereochemistries (namely $\alpha$ and $\beta$ ); and glycans exist as branching and linear structures (Figure 1). For systems where the biological pathways are fairly well characterized such as mammalian $\mathrm{N}$-glycans, high throughput analytical strategies have been developed for their characterization and even quantification. These strategies often rely on (ultra)high-performance liquid chromatography to separate the swathes of glycans and their glycoforms present in a biological matrix. Detection is facilitated by incorporation of a tag '(commonly to the reducing-terminus) prior to chromatography and fluorescence/UV detection and/or mass spectrometry (MS). Retention times calibrated to an internal standard (often dextran ladders) can be compared to known structures enabling identification.[10-12]

For less studied systems, combinations of analytical strategies are often employed including NMR, LC, and tandem mass spectrometry (MS/MS).[13] LC is often used solely to separate coexisting glycoforms. Although, structural information can be inferred by monitoring retention time shifts upon application of known specific glycosidase cocktails.[12] NMR offers the greatest level of atomistic structural information, although typically requires larger amounts of purified glycans, which is often difficult to obtain from biological samples.[14-16] As a result, MS(/MS) techniques have risen to the forefront, since they can detect low amounts of substrate, can be readily coupled to LC or hybridized with gas-phase separation techniques such as ion mobility spectrometry (IMS) that separate ions by their rotationally averaged collision-cross section (CCS), are rapid and can provide various levels of structural information.[17-19] During this time, a plethora of fragmentation techniques and hybridized versions have been studied on a variety of glycans and glycoconjugates. Routinely, glycans are fragmented by collision-induced dissociation (CID) producing glycosidic fragments (B-, Z-, C- and Y-) and cross-ring fragments (i,j $\mathrm{A}$ - and $\mathrm{i}, \mathrm{j} X-)$ depending precisely where dissociation occurs.[7] Recently, tunable infrared multiple photon dissociation (IRMPD) and related gas-phase IR techniques have risen to the forefront of interest given their ability to potentially provide unique fingerprints for isomeric glycans and/or fine structural information especially when combined with theoretical calculations, often inaccessible by MS alone (Figure $1 \mathrm{~b}$ and 1c).[20-25] This review intends to specifically cover recent work in the area of gas-phase IR spectroscopy coupled to MS for the analysis of glycans.

\section{IRMPD and gas-phase spectroscopy}

Gas-phase IR spectroscopy-MS of carbohydrates is a growing area of research, in part due to the increased accessibility of tuneable IR lasers, but also appreciation of the wealth of information and subtle differences observed in features within vibrational spectra of glycans;[18,26] perhaps unsurprising to chemists familiar with diagnostic $\mathrm{N}-\mathrm{H}$ and $\mathrm{O}-\mathrm{H}$ vibrational modes within conventional IR spectra given glycans contain a plethora of these linkages. These features depend on numerous structural aspects including the charge, composition, regio- and stereo-chemistry of the glycosidic linkage, the topology, ring size and finally the 3D structure. This 
provides complementary information to MS where it is extremely challenging to ascertain precisely stereochemical and conformational features. Unlike conventional spectroscopic techniques that measure the direct absorption of radiation upon interaction with the target sample, molecular ion beams/clouds generated within a mass spectrometer normally have insufficient analyte density to be able to measure the difference in intensity between the incident and transmitted light. Instead, a change within the analyte ions structure (such as dissociation) upon absorption of IR photons is measured (referred to as IR action spectroscopy). Classically, ion clouds are held in a mass analyzer such as a quadrupole or linear ion trap, allowing the user to control irradiation times. The efficiency of light absorption depends on the frequency of the incident light as well as the analytes atomic structure(s). With tunable IR lasers, a spectral range can be scanned and a gas-phase vibrational spectrum of an $\mathrm{m} / \mathrm{z}$ selected ion can be constructed. As eluded to, the dissociation yield of a trapped ion upon absorbing multiple photons (i.e. InfraRed multiple photon dissociation, IRMPD) as a function of the IR wavelength is one way to generate gasphase spectra.[26,27] A laser powerful enough to produce sequential absorption of numerous photons is necessary to overcome the analyte ions dissociation threshold, since each IR photon is low energy. The specific mechanisms of IR absorption and resultant dissociation are beyond the scope of this review but have previously been detailed.[26,27] As exemplified in this review, closely related isomers have distinctive IR fingerprints (note: all IR spectra presented in the figures are experimental data) and this property can be exploited to identify unknown glycans by comparison with a library of experimental IR spectra of standards. For such analytical purpose, theoretical modelling is not necessary. However, further structural information such as the 3D conformation can be obtained by combining experimental and theoretical approaches.[27] A typical routine for the production of theoretical IR spectra is carried out in two phases: firstly, a large number of candidate geometries are produced by molecular dynamics. At this stage, the current computational performance allows addressing large polysaccharides. The geometries are then refined by quantum approaches (typically Density Functional Theory) to produce high precision geometries and relative energies, at the cost of more severe size limitations: all-atom quantum approaches can only deal with small oligosaccharides. Secondly, the IR spectrum of the equilibrium structures are generated. This is typically done at high level of calculation (i.e. a large basis set) in the harmonic approximation, in order to generate high precision IR frequencies (within a few percent of the experimental frequencies). Finally, the modelled IR spectra are compared with experimental IR data to identify the 3D conformation adopted by the species of interest in the experimental condition.

Most early work on IRMPD-MS of glycans focussed on differences in the generated fragments upon irradiation at a series of defined narrow wavelengths as groups were limited to accessible $\mathrm{CO}_{2}$ lasers.[28] Nowadays, benchtop OPO/OPA lasers can scan a larger range (normally around $3 \mu \mathrm{m}$, where $\mathrm{OH}$ groups typically strongly absorb photons), however are still too low power to access the entire spectra range. These ranges became accessible by the development of free electron lasers (FEL) such as FELIX.[26,29] A variety of glycan families have now been analyzed by gas-phase IR spectroscopy including sialic acid (Neu5Ac) 
regioisomers,[8] hexosamine sulfate and phosphate isomers,[30,31] Oglycopeptides,[32] disaccharide regioisomers,[33] pyranose/furanose isomers,[34] chitooligosaccharides with differing $\mathrm{N}$-acetylation patterns[35] and hexuronic acid

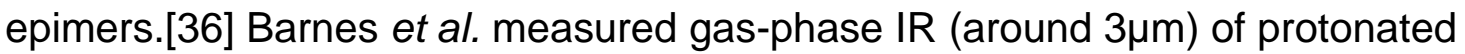
GlcNAc, GalNAc, and ManNAc and their $\alpha / \beta$ 1-O-Me counterparts which locks the anomeric configuration. All these $\alpha / \beta$ 1-O-Me HexNAcs exist as ${ }^{4} \mathrm{C}_{1}$ chair conformation stabilized by a network of hydrogen bonds around the ring hydroxyl groups, although some other low energy conformations that differed by features such as the orientation of the acetyl group and $\mathrm{H}-\mathrm{C}(6)-\mathrm{O}(6)-\mathrm{H}$ dihedral angle contributed to variations in each respective IR spectra. The free HexNAcs were all observed to be ${ }^{4} \mathrm{C}_{1}$ chair conformations as well. Interestingly, diagnostic features from the anomerically pure HexNAcs could be used to ascertain which features from the naturally occurring mixtures were diagnostic for each anomer, which could be powerful in determining ratios of these species.[20] Tan et al. also studied these three HexNAc epimers, but as lithium adducts and, in this case, found again all epimers were distinguishable by $\mathrm{N}-\mathrm{H}$ and $\mathrm{O}-\mathrm{H}$ stretching modes and GlcNAc and GalNAc still form ${ }^{4} \mathrm{C}_{1}$ chair conformation, however features in the vibrational spectrum suggest ManNAc is ${ }^{1} \mathrm{C}_{4}$.[37] This highlights the difference in structure that can occur from merely altering the adduct, which could alter the ions chemistry (e.g. preferred fragmentation pathways). Diagnostic spectral features have also been shown to be capable of discerning $\alpha 2,3$ versus $\alpha 2,6$ sialic acid containing saccharides. a2,3-linked sialic acids displayed a broad unresolved spectrum irrespective of the attached glycan or the adduct compared to the $\alpha 2,6$ regioisomers which were always very well resolved.[8] Differentiation of these naturally occurring motifs is important considering their differential interactions. One such example is influenza virus infectivity that depend on the specificity of influenza hemagglutinins (HA) binding. Avian influenza viruses more prominently bind Neu5Aca2,3Galß1,4GlcNAc, whereas human and swine influenza preferentially bind Neu5Aca2,6Galß1,4GlcNAc. One or two amino acid mutations of the avian HA can drastically alter their preference towards Neu5Aca2,6Gal (human-like), highlighting the delicate nature of glycan-recognition. These mutations are likely a prerequisite for human epidemic strains.[38,39] In reality, various strains of viral Has display more fine-tuned specificities to similar epitopes including fucosylation, sulfation and sialylation at positions 2 (Gal) and3 (GlcNAc).[39] One concern of tunable gas-phase IR measurements used to be that their longer acquisition times (often 10's of minutes) makes it unsuitable for liquid workflows (peak widths typically $<1 \mathrm{~min}$ ), which are ubiquitous within glycomics labs for separating mixtures of glycans, quantifying their abundances and/or providing structural identification.[10-12] However, recent technical developments are bringing this time down to minutes, and using a stop flow approach where when a LC peak is expected the flow is substantially lowered, the first online LC-MS-IRMPD experiment was able to be performed.[33] 
(a)

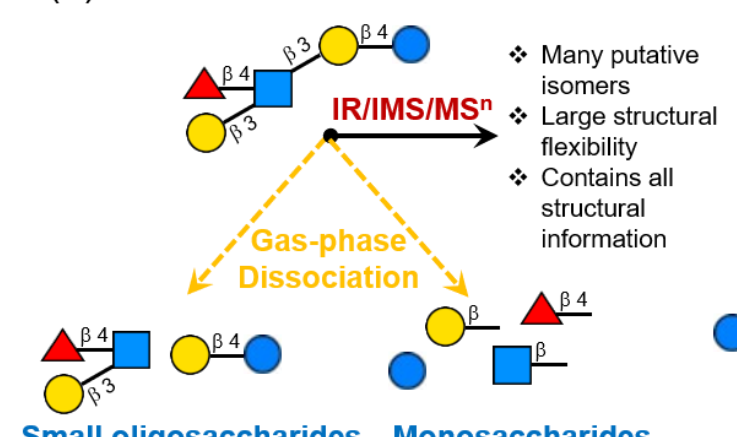

Small oligosaccharides Monosaccharides

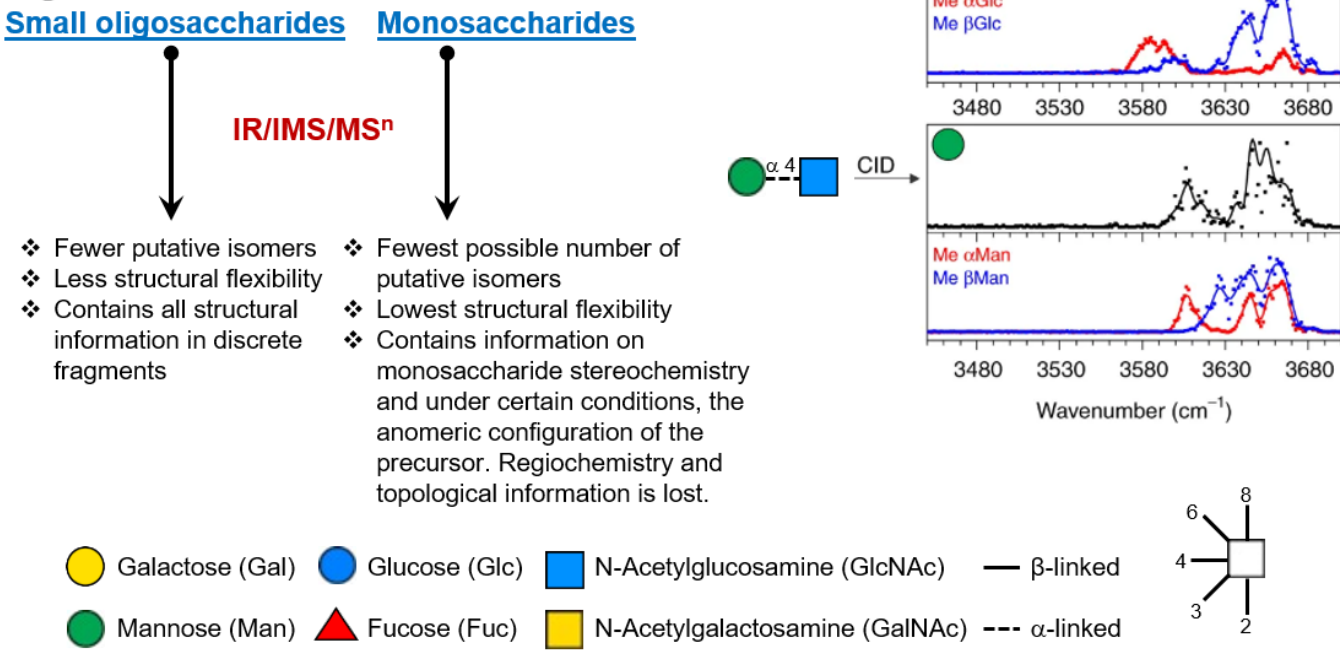

Figure 2. Benefits of analyzing the precursor as well as small oligosaccharide and monosaccharide product ions by gas-phase IR spectroscopy (although this extends to other approaches such as MS ${ }^{n}$ and IMS) (a). The sheer number of isomers of a precursor make unambiguous characterization of an unknown challenging. Also, for IR-MS, the conformational flexibility makes recording 'useful' well resolved spectra challenging. As you go down to smaller oligosaccharides, the number of putative isomers drastically reduces and from reduced conformational flexibility, IR-MS spectra are easier to acquire. This is beneficial for fingerprint database approaches. The topology of the glycan must be built up from fragments, therefore it is imperative that a complementary sequence is produced. Monosaccharide product ions are simplest in terms of flexibility and putative isomers, but connectivity information is lost. Analysis times also increase for studying multiple fragments. Example gas-phase IR spectra of the non-reducing C-ions generated from collision-induced dissociation (CID) of three disaccharides (black trace) (b). Below each spectrum are IR traces of the respective $\alpha-/ \beta-1-O M e$ hexose anomer standards ( $\alpha$ is red trace and $\beta$ is blue trace). Features in the equivalent fragment and appropriate methyl-anomer standard (i.e. mannose product ion derived from Mana1,4GIcNAc displayed similar features with a1-OMe Man), suggesting memory of the anomeric configuration of the precursor within the product ion.[ reproduced with permission from Ref 40] Oligosaccharide structures are shown in representative SNFG nomenclature.[9]

The examples above dealt with IRMPD of precursor ions. However, in ion-trapping devices it is also possible to perform IRMPD on CID generated product ions. This is potentially powerful for a number of reasons (Figure 2a).[13] Firstly, to deconvolute spectra; most gas-phase IR measurements around 300K are limited to small oligosaccharides as beyond these sizes, spectral features begin to overlap resulting in extremely broad unresolvable features.[35] For example, Renois-Predelus et al. found that mixtures of glycosaminoglycans (GAGs) chondroitin sulfate and dermatan sulfate di-/tetra-saccharides that differed by sulfation pattern (GalNAc4S versus GalNAc6S) result in poorly resolved, inseparable spectra, MS $^{n}$ (CID) and subsequent IRMPD of shorter diagnostic product ions, facilitates observation of key characteristic IR spectral features that are lost in the precursor.[41] Given their roles 
in numerous biological process,[6] analytical methods like this that are capable of differentiating GAGs are highly sought after.[41] Especially since, their identification poses a significant analytical challenge considering that there are subtle structural differences within and between each GAGs family including stereochemistry of the HexNAc (GlcNAc vs GalNac), hexuronic acid (GlcA vs IdoA), presence of HexN, regiochemistry of the linkages within the polysaccharides and presence and regiochemistry of sulfation.

Larger structures beyond trisaccharides also become more computationally challenging to model and generate useful theoretical spectra. Diagnostic fragments of interest could even be selectively probed whilst the rest of the molecule 'discarded' simplifying analysis. Spectral library databases would also benefit, since there are fewer possible isomers of short di-, tri- and tetra-saccharides compared to larger carbohydrates. In theory, most of a glycans sequence information can be 'rebuilt' from disaccharide fragments, assuming all disaccharide fragments are generated which unfortunately is not always the case. This is especially true given the growing amount of evidence that for cationized glycans, memory of the stereochemistry of the precursors glycosidic bond is retained in C- and surprisingly B-product ions after CID.[21,40,42,43] This was first observed by ion mobility spectrometry (IMS)-MS of a sodiated pentasaccharide,[42] although was studied in greater detail with lithiated disaccharide anomers.[21] Combined gas-phase IR, IMS and molecular modelling suggested that for diglucoside anomers, C-ions were $\alpha$ glucopyranosides or $\beta$-glucopyranosides depending on if they were derived from the $\alpha$ - or $\beta$-glucose linkage regardless of the regiochemistry.[21] At higher energies conversion of the $\alpha$ - to the $\beta$-glucopyranose structure were observed by IMS. B-ions were observed to exist as potentially mixtures of multiple structures making the precise assignment challenging without combined gas-phase separation. Differences in the IMS spectra of ring-size isomers (Galpa, Galp $\beta$ and Galf $\beta$ ) were also observed suggesting their memory may also be conserved.[21] Schindler et al. recorded the gas-phase IR spectra for multiple glycan anomers standards (1-OMe monosaccharides which locks reducing anomeric configuration by preventing mutorotation) and found them to be different (red and blue traces in Figure $2 b$ ). Unique features in the IRMPD spectra of each anomer standard extended to the natural free-monosaccharides precursors and more importantly CID fragments, suggesting firstly that again there is an anomeric memory and secondly this small library could be used to provide the composition and anomeric configuration of a monosaccharide fragment derived from a variety of precursors.[40] Given that biological interactions between glycans and other molecules is often highly specific to glycan structures and glycan biosynthesis is not a template-driven process, de novo methods that aid identifying the complete stereochemical (as well as regiochemical) structural motifs within glycans are crucial. This can lead to the development of glycan-based therapeutics and diagnostics.[44]

\section{Cryogenic gas-phase IR spectroscopy-MS}

Rather than dissociation of the analyte, precursors can also be tagged with clusters of inert "messenger" atoms or molecules such as $\mathrm{N}_{2}, \mathrm{Ar}$ or $\mathrm{H}_{2}$ respectively via weak ion-induced dipole interactions and dissociation of this complex monitored by MS.[26,27] The messengers very poorly absorb IR radiation, are polarizable enough to adhere to the analyte ion and ideally do not perturb the precursors conformation. As the interaction is weak, few IR photons need to be absorbed to 
break the intermolecular interaction causing less ion-heating which impacts upon the resolution of the IR spectra. Care must be taken using $\mathrm{H}_{2}$ as the messenger though, as it has been reported to influence the frequencies of some vibrational bands within protonated glycine.[45] This methodology has recently been exploited in cryogenicIR spectroscopy. Ben Faleh et al. have developed a remarkable custom-built instrument that combines ultrahigh-resolution IMS and cryogenic messenger-tagged IR spectroscopy (Figure 3a).[23] Produced ions are introduced into a SLIM (structures for lossless ion manipulation) device which uses travelling-wave IMS (TWIMS) to move ions through a serpentine path $(\sim 1.8 \mathrm{~m})$ in the presence of an inert buffer gas and separates ions based on differences in their CCS. Ions could be cycled back into the device improving IMS resolution at the cost of sensitivity. Ions whose CCS differed by as little as $0.2 \%$ could be separated. An electrostatic bender and quadrupole could be used to selectively isolate ions with specific mobilities and $\mathrm{m} / \mathrm{z}$ respectively, prior to introduction into the cryogenic ion trap. The cryogenic ion trap is cooled to $40 \mathrm{~K}$ in the presence of a He: $\mathrm{N}_{2}$ mix (90:10) during which the messenger $\mathrm{N}_{2}$ condenses onto the analyte. This trapped complex is then probed by a variable IR pulse, which boils off the weekly adhered $\mathrm{N}_{2}$ molecule when the structure is in resonance with the IR wavelength. Each glycan studied produced 2 resolvable IMS peaks suggesting each form are either distinct $\alpha$-/ $\beta$-anomers or less likely, different conformers of the same structure (Figure $3 b$ ). Each peak could be selectively probed by cryogenic gas-phase IR spectroscopy producing separable highly-resolved unique spectra for a series of epimeric disaccharides even from a mixture of them (Figure 3c).[23] This set-up expanded upon similar previous work on pairs of isomeric human milk oligosaccharides (HMOs) LNnT (Lacto-N-neotetraose) and LNT (Lacto-N-tetraose), 2'-FL and 3-FL and LNDFH I and LNDFH II tagged with $\mathrm{N}_{2}$ and also GAGs chondroitin sulfate and heparin sulfate isomers that differed by sulfation position tagged with $\mathrm{H}_{2}$. These isomers were all distinguishable by their IR spectra, which again were better resolved and separated compared to their room temperature counterparts,[30,46] however the $2 \mathrm{~m}$ long drift tube (using drift tube IMS, DTIMS separation rather than TWIMS) was insufficient to provide baseline mobility separation of all structures.[46,47] The role of HMOs are not fully understood, but are believed to provide key benefits towards infant health including shaping the gut microflora, acting as decoys for pathogenic microbes and regulators of immune response as well as other things. [48] Human galectins, GBPs implicated in immune regulation, have recently been reported to bind to these sugars, with some particularly sensitive to slight structural differences such as hGal-7 that prefers to adhere to the LNT tetrasaccharide motif and displays much lower affinity towards $\mathrm{LNnT}$; the single difference of regiochemistry of the terminal galactose $(\beta 1,3$ vs $\beta 1,4$, respectively).[48] 


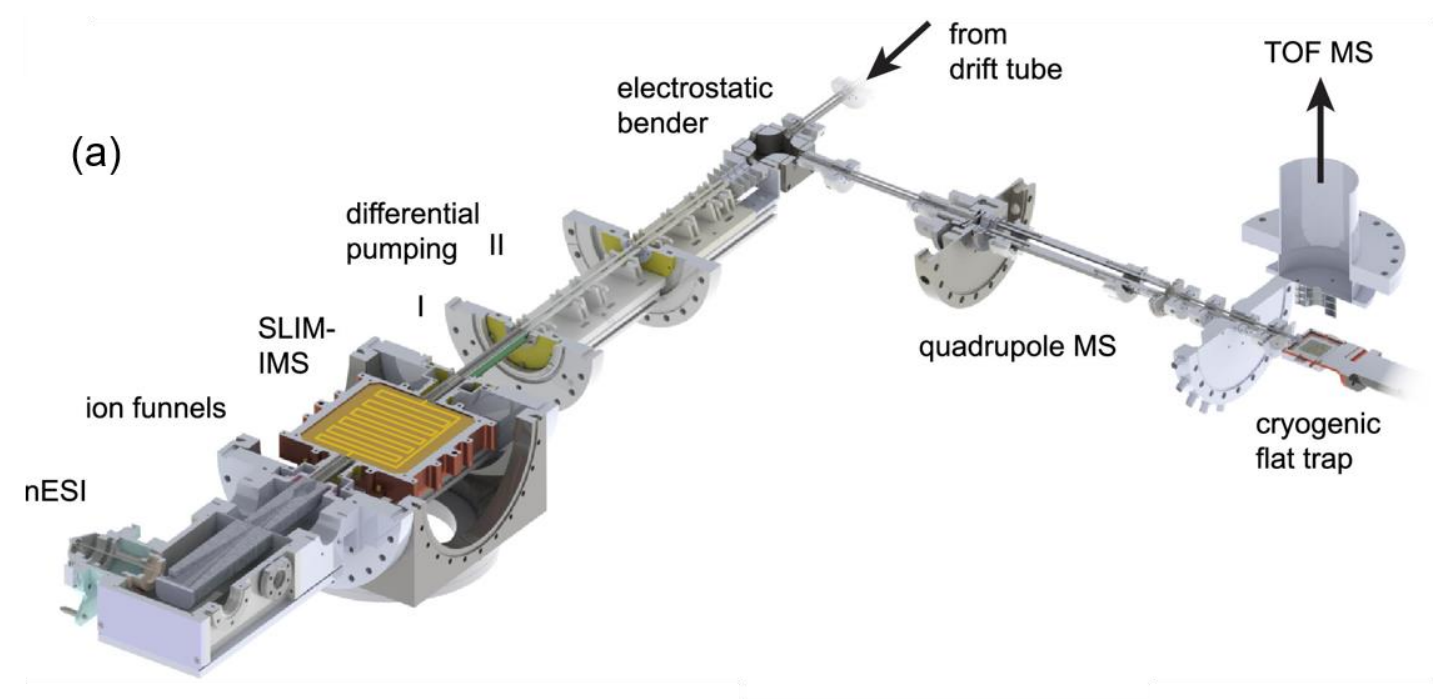

(b) $\mathrm{O}_{\bar{\beta} 4}$

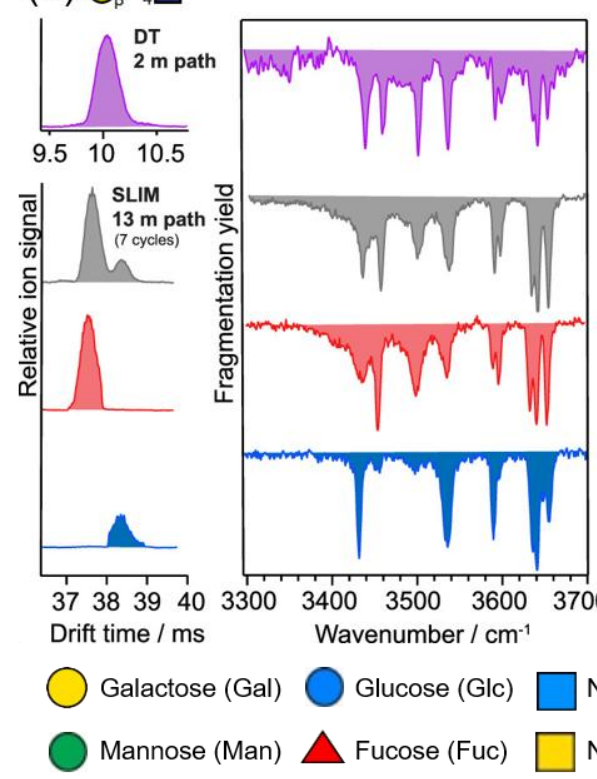

(c)

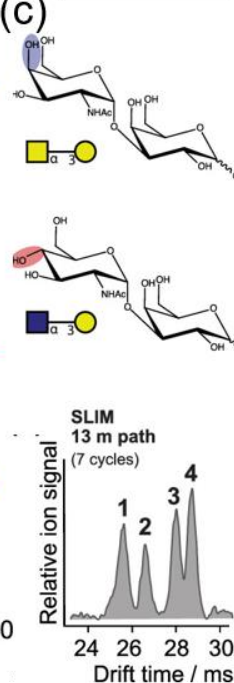

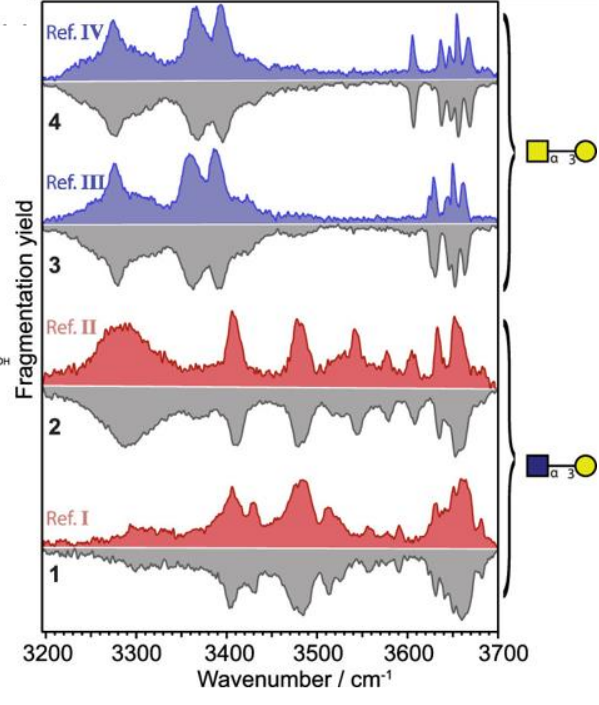

N-Acetylglucosamine (GlcNAc)

Figure 3. Overview of the instrument developed by Ben Faleh et al. for cryogenic gas-phase messenger-tagging IR spectroscopy (a). Ions produced by nano-electrospray ionization (nESI) are guided into a $1.8 \mathrm{~m}$ serpentine travelling-wave ion mobility spectrometry (TWIMS) device (structures for lossless ion manipulations, SLIM), where ions can be separated by their mobility through and inert buffer gas (this is related to the ions rotationally averaged collision-cross section (CCS)). lon could be reinjected into the SLIM device for multiple cycles, improving IMS resolution at the cost of sensitivity. lons could be mobility and then $\mathrm{m} / \mathrm{z}$ isolated prior to entering the cryogenic ion trap (40 K). Ions are cooled where an inert gas (such as $\mathrm{N}_{2}$ ) condenses to the analyte ion and acts as a 'messenger'. This messenger boils off when the structures vibrational modes are in resonance with the wavelength of a tunable IR laser from which a gas-phase IR spectrum can be plotted. Also shown are the arrival time distributions (ATDs) of Galß1,4GIcNAc (b) on a lower resolution drift tube (purple, left side) compared to the SLIM device (gray, left side), where presumably the two coexisting anomers are separated. ATDs are shown with respective mobility isolation of each anomer (red and blue, left side). On the right are the cryogenic IR spectra of each of these respective species particularly noticeable are the bands are well-resolved and the difference in spectra between the anomers. A mixture of two epimeric disaccharides (c) are also studied which are baseline resolved in the ATD (including the coexisting anomers) and each species separately probed by cryogenic gas-phase IR and compared to this pure reference standard.[ reproduced with permission from Ref 23] 
Two-laser double-resonance IR-IR spectroscopy methodologies have also been found to be useful for studying specific glycan conformers. Traditionally this set-up involves an initial high fluence IR pump-laser pulse fixed at a wavenumber specific for a transition within the target conformer. Shortly after, these ions are then irradiated with a tunable IR probe-laser pulse. When a structure is resonant with the pump-laser and scanning probe-laser, a dip in the photofragmentation yield occurs (often referred to as IR-IR ion-dip spectrums).[22,24] Other strategies of performing double resonance IR-IR have been applied to glycans that are not detailed here.[22] Voss et al. were able to use double resonance IR-IR to monitor and determine conformational families of sodiated glucose using $\mathrm{D}_{2}$ as the messenger.[22] Similarly, Scutelnic and Rizzo combined IR-IR double-resonance spectroscopy in a cryogenic ion trap with ${ }^{18} \mathrm{O}$ labelling and quantum mechanical computations to determine the conformational families of protonated glucosamine (GlcN).[24] The reducing-end hydroxyl group can be readily exchanged with ${ }^{18} \mathrm{O}-\mathrm{H}$ merely by dissolution of the glycan in ${ }^{18} \mathrm{OH}_{2}$ and waiting for 48 hours. Monitoring the bands that shift in the IR spectrum of the isotopically unlabelled versus labelled saccharide, facilitates easy identification of bands associated with the reducing hydroxyl group. Two bands were observed to shift suggesting the presence of at least two conformer families. IR-IR spectroscopy in fact found a third conformation with features similar to another conformer (suggesting similar hydrogen bonding networks between the two conformers). Calculations found these to be ${ }^{1} \mathrm{C}_{4} \alpha-\mathrm{GlcN},{ }^{4} \mathrm{C}_{1} \alpha-\mathrm{GlcN}$ and ${ }^{4} \mathrm{C}_{1} \beta-\mathrm{GlcN}$, with the latter two being the similar conformers. Pleasingly, the $\alpha-\beta$ - ratios were found to be comparable to those measured by polarimetry, NMR and microchip capillary electrophoresis in the solution-phase.[24]

Recently, an alternative cryogenic IR strategy has been developed by Mucha et al. using superfluid helium droplets to cool down glycan analyte ions.[25] Ion produces by $\mathrm{nESI}$ are $\mathrm{m} / \mathrm{z}$ selected and accumulated in a linear ion tap. Superfluid helium droplets $\left(\sim 10^{5}\right.$ atoms $)$ are guided through the trap where they pick up these ions. These droplets rapidly cool the ions to the internal temperature of the droplet $(0.37 \mathrm{~K})$. Ions are ejected from the droplet by IR irradiation, when the wavelength is in resonance with a vibrational mode of the analyte. Scanning IR wavelength enables plotting of an ultracold IR spectra of a particular molecular ion of interest. Using this, they studied a series of isomeric mono-/di- and tri-saccharides as well as isomeric pairs of biologically relevant tetrasaccharides $\mathrm{Le}^{\mathrm{b}}$ and $\mathrm{Le}^{\mathrm{y}}$ and $\mathrm{LNnT}$ and LNT (Figure 4), many of which differed by combinations of the stereo- and regiochemistry of the glycosidic bonds and some by monosaccharide composition. Some of these were reducing-end labelled with an amino-alkyl moiety promoting protonation and locking the reducing-end stereochemistry, whereas the free-glycans were studied as sodium adducts. Spectra displayed unprecedented resolving power compared to $300 \mathrm{~K}$ measurements, and in addition, each spectrum was a unique fingerprint of that glycan. This facilitates a glycan characterization approach based upon databases of these spectra. Alternatively, features between a reference spectrum and an unknown could be compared to facilitate identification of structural features in the unknown. As mentioned previously, theoretical IR spectra of energy minimized structures can be generated and compared to acquired spectra improving confidence in structural assignments.[25] 
(a)

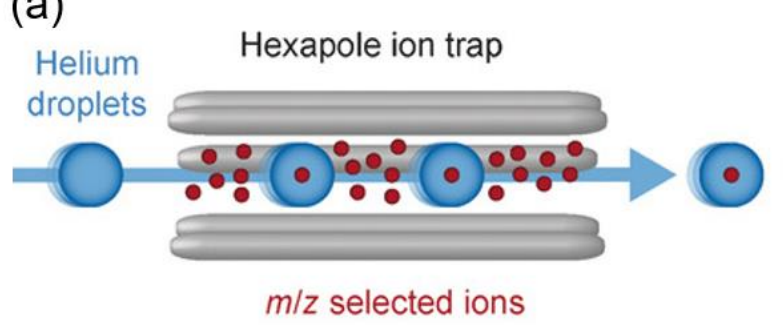

(b)
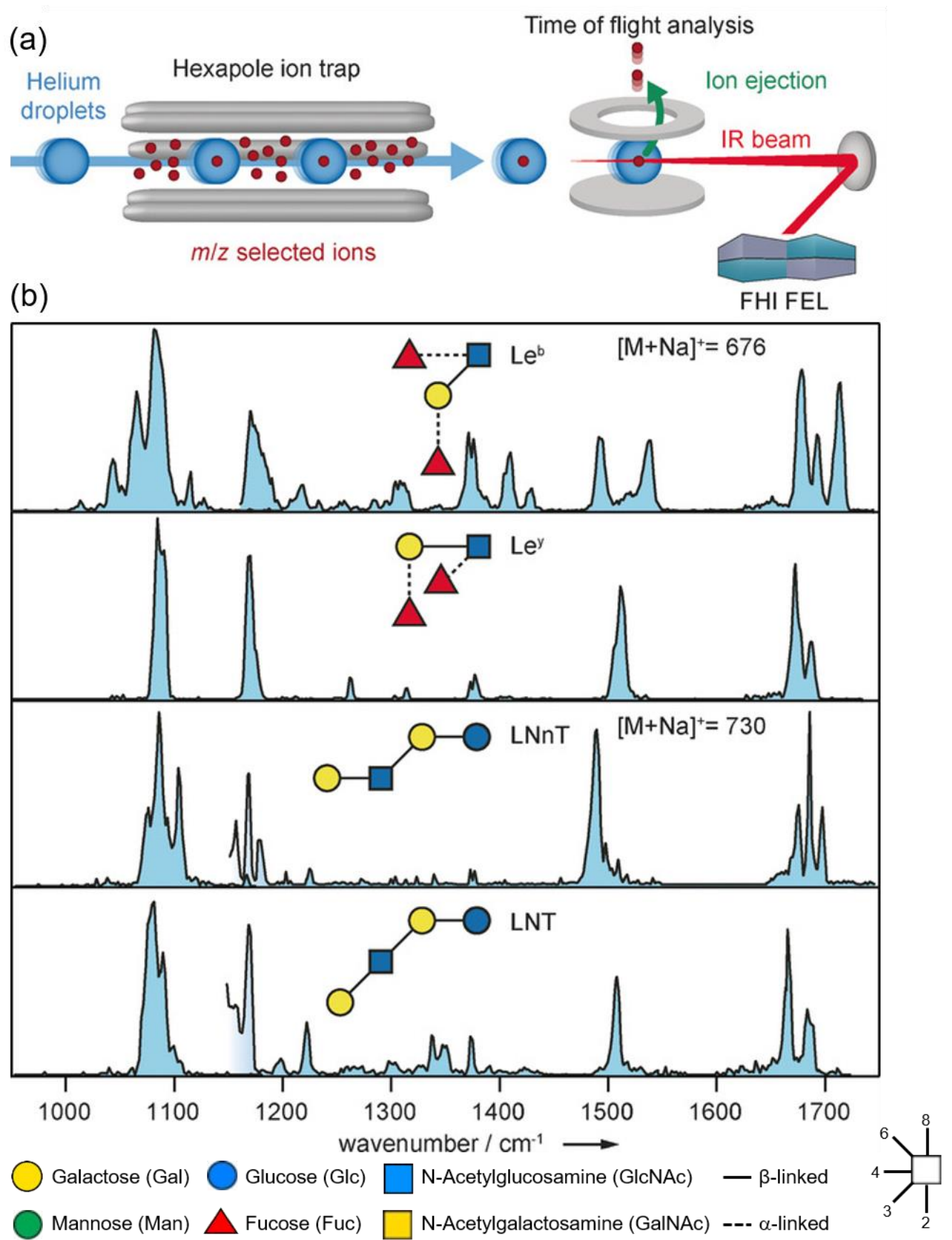

Figure 4. Scheme depicting the superfluid helium droplet set-up used by Mucha et al. (a) Gas-phase ions are $\mathrm{m} / \mathrm{z}$ selected and accumulated inside and ion trap. Superfluid helium droplets are guided through the trap where they pick-up these ions, and subsequently cool them down to $0.37 \mathrm{~K}$. The droplets are irradiated with a defined wavelength from a variable IR laser, where the ions are ejected when they are in resonance with the incoming laser beam. Well resolved IR spectra of isomeric sodiated tetrasaccharide pairs $\mathrm{Le}^{\mathrm{b}}$ and $\mathrm{le}^{\mathrm{y}}$ and LNnT and LNT are shown, highlighting that these isomers could readily be distinguished (b). [ reproduced with permission from Ref 25]

\section{Conclusions and future outlooks}

Gas-phase IR-MS ${ }^{(n)}$ combined with molecular modelling and theoretical calculations has been shown to be capable of differentiating a number of isomeric glycans, even those that differ only by the stereochemistry at a single position.[23,40,47] Currently, these approaches are limited to relatively shorter carbohydrates, since as the size of these ions get larger, the more congested these 
vibrational spectra become to the point where likely only an undiagnostic broad mess of overlapping signals will be observed. Efforts in cryogenic gas-phase IR spectroscopy significantly improve IR resolution through cooling the 'hot' ions and have as a result enabled larger saccharides to be studied and differentiated (around hexasaccharides). These unique IR fingerprints could be housed within databases and features compared to unknowns to either identify the entire sequence, or in the case of a compete unknown possibly identify some structural features. It's still yet to be shown if these approaches will be able extend to even larger carbohydrates or even polysaccharides.

An alternative approach is based on fragmentation, i.e. analysis of smaller product ions rather than the intact precursor ions. This reduces the complexity of the spectrum admittedly at the cost of duty cycle since multiple product ions would have to be screened compared to a single precursor. However, with some prior knowledge of the structure (e.g. from the MS/MS spectrum) and fragmentation pathways, certain diagnostic ions could be targeted. An added benefit of this approach is that reference libraries of di-/tri-/tetra-saccharides would be smaller than one of all its precursors (consider the number of potential isomers of disaccharides compared to a hexasaccharide for example). At the moment, this approach relies on complementary sequence of product ions forming, and a more thorough understanding of fragmentation chemistry of the precursor ion and the fragmentation techniques is needed. The integration of information from multiple analytical areas such as LC, IMS, NMR and enzymatic digestion is currently one of the most promising strategies towards glycan structural assignment workflows and will require greater cross-discipline collaborations, including comprehensive databases and bioinformatics tools. Indeed, recent instrumentation hybridizing IMS-IR-MS, has already shown successes in providing fast and high-quality structural information on glycan sequences for several glycan families.

\section{Acknowledgements}

This work was supported by ERC (788231-ProgrES-ERC-2017-ADG) EU (CarboMet 737395) BBSRC (IBCarb BB/L013762/1; BB/M027791/1; BB/M02903411; BB/M028836/1) to C.J.G and S.L.F.

\section{References}

1. Varki A: Biological roles of glycans. Glycobiology 2017, 27:3-49.

2. National Research Council (US) Committee on Assessing the Importance and Impact of Glycomics and Glycosciences: Transforming Glycoscience: A Roadmap for the Future. Washington, DC: The National Academies Press; 2012.

3. Ho W-L, Hsu W-M, Huang M-C, Kadomatsu K, Nakagawara A: Protein glycosylation in cancers and its potential therapeutic applications in neuroblastoma. Journal of Hematology \& Oncology 2016, 9:100.

4. Yoshida A, Kobayashi K, Manya H, Taniguchi K, Kano H, Mizuno M, Inazu T, Mitsuhashi H, Takahashi S, Takeuchi M, et al.: Muscular Dystrophy and Neuronal Migration Disorder 
Caused by Mutations in a Glycosyltransferase, POMGnT1. Developmental Cell 2001, 1:717724.

5. Jaeken J, Matthijs G: Congenital Disorders of Glycosylation: A Rapidly Expanding Disease Family. Annual Review of Genomics and Human Genetics 2007, 8:261-278.

6. Varki A, Cummings RD, Esko JD, Stanley P, Hart GW, Aebi M, Darvill AG, Kinoshita T, Packer NH, Prestegard JH, et al.: Essentials of Glycobiology edn 3rd: Cold Spring Harbor (NY): Cold Spring Harbor Laboratory Press; 2015-2017.

7. Domon $B$, Costello $C E$ : A systematic nomenclature for carbohydrate fragmentations in FABMS/MS spectra of glycoconjugates. Glycoconjugate Journal 1988, 5:397-409.

8. Depraz Depland A, Renois-Predelus G, Schindler B, Compagnon I: Identification of sialic acid linkage isomers in glycans using coupled InfraRed Multiple Photon Dissociation (IRMPD) spectroscopy and mass spectrometry. International Journal of Mass Spectrometry 2018, 434:65-69.

* $\alpha 2,3$ and $\alpha 2,6$ sialic acid regioisomers that both occur naturally were distinguishable by consistent diagnostic features within their IR spectra, despite them producing very similar MS/MS spectra.

9. Varki A, Cummings RD, Aebi M, Packer NH, Seeberger PH, Esko JD, Stanley P, Hart G, Darvill A, Kinoshita T, et al.: Symbol Nomenclature for Graphical Representations of Glycans. Glycobiology 2015, 25:1323-1324.

10. Campbell MP, Royle L, Radcliffe CM, Dwek RA, Rudd PM: GlycoBase and autoGU: tools for HPLCbased glycan analysis. Bioinformatics 2008, 24:1214-1216.

11. Zhao S, Walsh I, Abrahams JL, Royle L, Nguyen-Khuong T, Spencer D, Fernandes DL, Packer NH, Rudd PM, Campbell MP: GlycoStore: a database of retention properties for glycan analysis. Bioinformatics 2018, 34:3231-3232.

12. Walsh I, Nguyen-Khuong T, Wongtrakul-Kish K, Tay SJ, Chew D, José T, Taron CH, Rudd PM: GlycanAnalyzer: software for automated interpretation of $\mathrm{N}$-glycan profiles after exoglycosidase digestions. Bioinformatics 2019, 35:688-690.

13. Gray CJ, Migas LG, Barran PE, Pagel K, Seeberger PH, Eyers CE, Boons G-J, Pohl NLB, Compagnon I, Widmalm G, et al.: Advancing Solutions to the Carbohydrate Sequencing Challenge. Journal of the American Chemical Society 2019.

14. Fontana $\mathrm{C}$, Kovacs $\mathrm{H}$, Widmalm $\mathrm{G}$ : NMR structure analysis of uniformly $13 \mathrm{C}$-labeled carbohydrates. Journal of Biomolecular NMR 2014, 59:95-110.

15. Yang MJ, d'Ortoli TA, Sawen E, Jana M, Widmalm G, MacKerell AD: Delineating the conformational flexibility of trisaccharides from NMR spectroscopy experiments and computer simulations. Physical Chemistry Chemical Physics 2016, 18:18776-18794.

16. Fontana C, Conde-Alvarez R, Stahle J, Holst O, Iriarte M, Zhao Y, Arce-Gorvel V, Hanniffy S, Gorvel JP, Moriyon I, et al.: Structural Studies of Lipopolysaccharide-defective Mutants from Brucella melitensis Identify a Core Oligosaccharide Critical in Virulence. Journal of Biological Chemistry 2016, 291:7727-7741.

17. Lanucara F, Holman SW, Gray CJ, Eyers CE: The power of ion mobility-mass spectrometry for structural characterization and the study of conformational dynamics. Nature Chemistry 2014, 6:281.

18. Mucha E, Stuckmann A, Marianski M, Struwe WB, Meijer G, Pagel K: In-depth structural analysis of glycans in the gas phase. Chemical Science 2019, 10:1272-1284.

19. Gray CJ, Thomas B, Upton R, Migas LG, Eyers CE, Barran PE, Flitsch SL: Applications of ion mobility mass spectrometry for high throughput, high resolution glycan analysis. Biochimica et Biophysica Acta (BBA) - General Subjects 2016, 1860:1688-1709.

20. Barnes L, Schindler B, Chambert S, Allouche A-R, Compagnon I: Conformational preferences of protonated $\mathrm{N}$-acetylated hexosamines probed by InfraRed Multiple Photon Dissociation 
(IRMPD) spectroscopy and ab initio calculations. International Journal of Mass Spectrometry 2017, 421:116-123.

*Interesting study of $\mathrm{N}$-acetylhexosamine anomer conformational families by combining IRMPD spectroscopy and $a b$ initio calculations.

21. Gray CJ, Schindler B, Migas LG, Pičmanová M, Allouche AR, Green AP, Mandal S, Motawia MS, Sánchez-Pérez R, Bjarnholt N, et al.: Bottom-Up Elucidation of Glycosidic Bond Stereochemistry. Analytical Chemistry 2017, 89:4540-4549.

** First evidence by ion mobility spectrometry, mass spectrometry, gas-phase IR spectroscopy and $a b$ inito calculations that certain glycosidic product ions retain the 'memory' of the precursor glycosidic linkage for lithiated (and probably sodiated) carbohydrates. They also provide evidence for the $\mathrm{C}$-ions of what structures occur.

22. Voss JM, Kregel SJ, Fischer KC, Garand E: IR-IR Conformation Specific Spectroscopy of $\mathrm{Na+(Glucose)}$ Adducts. Journal of The American Society for Mass Spectrometry 2018, 29:4250.

** One of few groups who have applied double-resonance IR-IR spectroscopy to selectively probe different carbohydrate gas-phase conformers. They use this method to study the various conformational families present within sodiated glucose ions.

23. Ben Faleh A, Warnke S, Rizzo TR: Combining Ultrahigh-Resolution Ion-Mobility Spectrometry with Cryogenic Infrared Spectroscopy for the Analysis of Glycan Mixtures. Analytical Chemistry 2019, 91:4876-4882.

** Combined gas-phase cryogenic infrared spectroscopy-MS with ultrahigh-resolution travellingwave ion-mobility spectrometry SLIM device. This enabled conformer and $\mathrm{m} / \mathrm{z}$ selection prior to probing with an IR laser. Anomers of epimeric disaccharides could be separated and selected for analysis. Each species produce unique very well resolved action IR spectra.

24. Scutelnic V, Rizzo TR: Cryogenic Ion Spectroscopy for Identification of Monosaccharide Anomers. The Journal of Physical Chemistry A 2019, 123:2815-2819.

* Nice application of double-resonance IR-IR spectroscopy, isotopic labelling and theoretical calculations to determine the major conformational families present in protonated glucosamine. Facile ${ }^{18} \mathrm{O}$-labelling of the reducing-end anomeric hydroxyl group enabled easy identification of bands associated with the reducing $\mathrm{O}-\mathrm{H}$ group and suggested a minimum number of conformational families present. Double-resonance IR-IR measurements found three conformers to be present differing by their chair conformation and anomeric configuration.

25. Mucha E, González Flórez Al, Marianski M, Thomas DA, Hoffmann W, Struwe WB, Hahm HS, Gewinner S, Schöllkopf W, Seeberger PH, et al.: Glycan Fingerprinting via Cold-Ion Infrared Spectroscopy. Angewandte Chemie International Edition 2017, 56:11248-11251.

** Use of superfluid helium droplets to cool down trapped glycan ions to $0.37 \mathrm{~K}$, prior to IR irradiation. Spectra were extremely well resolved, producing unique fingerprints for multiple isomeric mono-/di-/tri-saccharides as well as isomeric pairs of biologically relevant tetrasaccharides including LNnT and LNT and $\mathrm{Le}^{\mathrm{b}}$ and $\mathrm{Le}^{\mathrm{y}}$.

26. Polfer NC: Infrared multiple photon dissociation spectroscopy of trapped ions. Chemical Society Reviews 2011, 40:2211-2221.

27. Rijs AM, Oomens J: IR Spectroscopic Techniques to Study Isolated Biomolecules. In Gas-Phase IR Spectroscopy and Structure of Biological Molecules. Edited by Rijs A, Oomens J: Springer, Cham; 2015:1-42. Topics in Current Chemistry, vol 364.] 
28. Polfer NC, Valle JJ, Moore DT, Oomens J, Eyler JR, Bendiak B: Differentiation of isomers by wavelength-tunable infrared multiple-photon dissociation-mass spectrometry: Application to glucose-containing disaccharides. Analytical Chemistry 2006, 78:670-679.

29. Oepts D, van der Meer AFG, van Amersfoort PW: The Free-Electron-Laser user facility FELIX. Infrared Physics \& Technology 1995, 36:297-308.

30. Schindler B, Barnes L, Gray CJ, Chambert S, Flitsch SL, Oomens J, Daniel R, Allouche AR, Compagnon I: IRMPD Spectroscopy Sheds New (Infrared) Light on the Sulfate Pattern of Carbohydrates. The Journal of Physical Chemistry A 2017, 121:2114-2120.

31. Schindler B, Joshi J, Allouche A-R, Simon D, Chambert S, Brites V, Gaigeot M-P, Compagnon I: Distinguishing isobaric phosphated and sulfated carbohydrates by coupling of mass spectrometry with gas phase vibrational spectroscopy. Physical Chemistry Chemical Physics 2014, 16:22131-22138.

32. Fukui K, Takahashi K: Infrared Multiple Photon Dissociation Spectroscopy and Computational Studies of O-Glycosylated Peptides. Analytical Chemistry 2012, 84:2188-2194.

33. Schindler B, Laloy-Borgna G, Barnes L, Allouche A-R, Bouju E, Dugas V, Demesmay C, Compagnon I: Online Separation and Identification of Isomers Using Infrared Multiple Photon Dissociation lon Spectroscopy Coupled to Liquid Chromatography: Application to the Analysis of Disaccharides Regio-Isomers and Monosaccharide Anomers. Analytical Chemistry 2018, 90:11741-11745.

* First example of online LC separation of carbohydrates coupled to tunable IRMPD-MS for carbohydrate analysis. The flow rate was significantly reduced when a carbohydrate peak was present, enabling IR acquisitions, which normally take minutes.

34. Schindler B, Legentil L, Allouche A-R, Ferrières V, Compagnon I: Spectroscopic diagnostic for the ring-size of carbohydrates in the gas phase: furanose and pyranose forms of GalNAc. Physical Chemistry Chemical Physics 2019, 21:12460-12467.

35. Wattjes J, Schindler B, Trombotto S, David L, Moerschbacher BM, Compagnon I: Discrimination of patterns of $\mathrm{N}$-acetylation in chitooligosaccharides by gas phase IR spectroscopy integrated to mass spectrometry. Pure and Applied Chemistry 2017, 89:1349-1357.

36. Schindler B, Renois-Predelus G, Bagdadi N, Melizi S, Barnes L, Chambert S, Allouche A-R, Compagnon I: MS/IR, a new MS-based hyphenated method for analysis of hexuronic acid epimers in glycosaminoglycans. Glycoconjugate Journal 2017, 34:421-425.

37. Tan Y, Zhao N, Liu J, Li P, Stedwell CN, Yu L, Polfer NC: Vibrational Signatures of Isomeric Lithiated N-acetyl-D-hexosamines by Gas-Phase Infrared Multiple-Photon Dissociation (IRMPD) Spectroscopy. Journal of The American Society for Mass Spectrometry 2017, 28:539-550.

38. Matrosovich M, Tuzikov A, Bovin N, Gambaryan A, Klimov A, Castrucci MR, Donatelli I, Kawaoka Y: Early Alterations of the Receptor-Binding Properties of H1, H2, and H3 Avian Influenza Virus Hemagglutinins after Their Introduction into Mammals. Journal of Virology 2000, 74:8502.

39. Stevens J, Blixt O, Glaser L, Taubenberger JK, Palese P, Paulson JC, Wilson IA: Glycan Microarray Analysis of the Hemagglutinins from Modern and Pandemic Influenza Viruses Reveals Different Receptor Specificities. Journal of Molecular Biology 2006, 355:1143-1155.

40. Schindler B, Barnes L, Renois G, Gray CJ, Chambert S, Fort S, Flitsch SL, Loison C, Allouche A-R, Compagnon I: Anomeric memory of the glycosidic bond upon fragmentation and its consequences for carbohydrate sequencing. Nature Communications 2017, 8:973.

** Expands on previous work by IMS and IR-MS, providing additional examples of the 'memory' of anomeric configuration within the product ions of saccharides. 
41. Renois-Predelus G, Schindler B, Compagnon I: Analysis of Sulfate Patterns in Glycosaminoglycan Oligosaccharides by MSn Coupled to Infrared Ion Spectroscopy: the Case of GalNAc4S and GaINAc6S. Journal of The American Society for Mass Spectrometry 2018, 29:1242-1249.

* Differentiation of major isomers present in chondroitin sulfate and dermatan sulfate by IRMPD.

42. Both P, Green AP, Gray CJ, Šardzík R, Voglmeir J, Fontana C, Austeri M, Rejzek M, Richardson D, Field RA, et al.: Addendum: Discrimination of epimeric glycans and glycopeptides using IMMS and its potential for carbohydrate sequencing. Nature Chemistry 2014, 6:368.

43. Mookherjee A, Uppal SS, Guttman M: Dissection of Fragmentation Pathways in Protonated NAcetylhexosamines. Analytical Chemistry 2018, 90:11883-11891.

44. Tommasone S, Allabush F, Tagger YK, Norman J, Köpf M, Tucker JHR, Mendes PM: The challenges of glycan recognition with natural and artificial receptors. Chemical Society Reviews 2019, 48:5488-5505.

45. Masson A, Williams ER, Rizzo TR: Molecular hydrogen messengers can lead to structural infidelity: A cautionary tale of protonated glycine. The Journal of Chemical Physics 2015, 143:104313.

46. Khanal N, Masellis C, Kamrath MZ, Clemmer DE, Rizzo TR: Glycosaminoglycan Analysis by Cryogenic Messenger-Tagging IR Spectroscopy Combined with IMS-MS. Analytical Chemistry 2017, 89:7601-7606.

*Cryogenic messenger-tagged IR spectroscopy produce better resolved and distinguishable spectra of chondroitin sulfate and heparan sulfate disaccharides, which differed by sulfation pattern, compared to similar studies by room temperature IRMPD.

47. Khanal N, Masellis C, Kamrath MZ, Clemmer DE, Rizzo TR: Cryogenic IR spectroscopy combined with ion mobility spectrometry for the analysis of human milk oligosaccharides. Analyst 2018, 143:1846-1852.

* Combining drift tube ion mobility spectrometry (DTIMS) with messenger-tagged cryogenic IR spectroscopy to study isomeric pairs of human milk oligosaccharides. DTIMS facilitated separation and mobility isolation of these isomeric species prior to IR irradiation. Each species was resolvable by IR although some were poorly resolved by DTIMS.

48. Noll AJ, Gourdine J-P, Yu Y, Lasanajak Y, Smith DF, Cummings RD: Galectins are human milk glycan receptors. Glycobiology 2016, 26:655-669. 